\title{
Hepatocellular Carcinoma: Current Management and Future Development-Improved Outcomes with Surgical Resection
}

\author{
Yoji Kishi, Kiyoshi Hasegawa, Yasuhiko Sugawara, and Norihiro Kokudo \\ Division of Surgery, Depatments of Hepatobiliary Pancreatic Surgery and Artificial Organ and Transplantation, \\ Graduate School of Medicine, The University of Tokyo, 7-3-1, Hongo, Bunkyo-ku, Tokyo 113-8655, Japan \\ Correspondence should be addressed to Kiyoshi Hasegawa, kihase-tky@umin.ac.jp
}

Received 15 February 2011; Revised 16 April 2011; Accepted 24 April 2011

Academic Editor: Daisuke Morioka

Copyright (๑) 2011 Yoji Kishi et al. This is an open access article distributed under the Creative Commons Attribution License, which permits unrestricted use, distribution, and reproduction in any medium, provided the original work is properly cited.

Currently, surgical resection is the treatment strategy offering the best long-term outcomes in patients with hepatocellular carcinoma (HCC). Especially for advanced HCC, surgical resection is the only strategy that is potentially curative, and the indications for surgical resection have expanded concomitantly with the technical advances in hepatectomy. A major problem is the high recurrence rate even after curative resection, especially in the remnant liver. Although repeat hepatectomy may prolong survival, the suitability may be limited due to multiple tumor recurrence or background liver cirrhosis. Multimodality approaches combining other local ablation or systemic therapy may help improve the prognosis. On the other hand, minimally invasive, or laparoscopic, hepatectomy has become popular over the last decade. Although the short-term safety and feasibility has been established, the long-term outcomes have not yet been adequately evaluated. Liver transplantation for HCC is also a possible option. Given the current situation of donor shortage, however, other local treatments should be considered as the first choice as long as liver function is maintained. Non-transplant treatment as a bridge to transplantation also helps in decreasing the risk of tumor progression or death during the waiting period. The optimal timing for transplantation after HCC recurrence remains to be investigated.

\section{Introduction}

Surgical resection is currently the standard option and treatment of first choice for hepatocellular carcinoma (HCC), given appropriate patient selection. The safety of surgical resection has been established over the last few decades, and the mortality rate after hepatic resection in experienced centers is less than $5 \%$. The indications for surgical resection have expanded even to advanced HCCs, as complicated hepatic resection can be safely performed owing to advances in the surgical techniques. At the same time, the minimally invasive approach, namely, laparoscopic hepatectomy, has also come to be increasingly advocated over the last decade. The safety and feasibility of laparoscopic hepatectomy after the learning curve period has been established in highvolume institutions, and the indications have been expanded from partial resection of benign tumors to major resection of malignant tumors, including HCCs. In this paper, the roles of aggressive hepatic resection for advanced HCC, the role of the recently advocated minimally invasive hepatectomy, the role of preoperative and postoperative adjuvant treatments, and the importance of a multimodality approach, including local ablation therapy, transarterial chemoembolization (TACE), and liver transplantation, are described.

\section{Expansion of the Indications for Surgical Resection}

Curative surgical resection is currently the only strategy for achieving a potentially satisfactory long-term outcome in patients with HCC and should, therefore, be the treatment of first choice as long as the tumor is judged to be resectable. Here, the term "resectability" is subjective and its definition varies according to the surgeons' skill. Several criteria to decide the indication for surgical resection based on the liver function and tumor status have been proposed. The method adopted for preoperative evaluation of liver function, which would determine the extent of resection of the liver, may vary 
among districts and institutions. In most Western countries, the presence/absence of portal hypertension is emphasized as an important criterion, which is estimated along with the Child-Pugh class, and is diagnosed based on the findings in hepatic venous pressure gradient, radiological images of splenomegaly and abdominal collaterals, thrombocytopenia (platelets $<100,000 / \mathrm{mm}^{3}$ ), and presence of esophagogastric varices $[1,2]$. One of the classical staging systems under the Barcelona Clinic Liver Cancer (BCLC) criteria included these criteria for the selection of appropriate treatments and recommended hepatic resection only for patients with a solitary HCC without portal hypertension [3]. Furthermore, this criterion is also included in the guideline for the treatment of HCC established by the American Association for the Study of Liver Diseases and European Association for the Study of the Liver [2,4]. In contrast, several studies reported the experiences of resection of multinodular HCCs or HCCs with portal hypertension yielding survival benefits, especially in patients where the background liver cirrhosis was classified as Child-Pugh class A [5, 6].

On the other hand, in Asian countries indocyanine green retention at 15 minutes (ICG-R15) is used for patient selection $[3,7,8]$. ICG-R15 is included in the preoperative evaluation of liver damage, along with determination of the presence/absence of ascites, serum bilirubin, serum albumin, and prothrombin time [9]. Recently published Japanese evidence-based clinical guidelines for the diagnosis and treatment of HCC propose surgical resection as the treatment of first choice for HCC patients with 3 or fewer nodules of any size categorized into liver damage of $\mathrm{A}$ or B [10]. This guideline recommend either surgical resection or local ablation therapy for early HCC [10]; however, it still remains under debate as to which of the two modalities might be more appropriate. Two randomized controlled trials (RCTs) comparing surgical resection and ablation therapy for small HCCs have been reported, and both yielded similar therapeutic results $[11,12]$. However, there were critical flaws in both studies, including an insufficient number of patients, imbalance in the background characteristics, and high conversion rates; therefore, the results of these two RCTs are invalid [13]. In 2010, Huang et al. reported the results of an RCT of hepatectomy and radiofrequency ablation (RFA) for patients with HCC fulfilling the Milan criteria (single tumor $\leq 5 \mathrm{~cm}$, or two or three tumors with none $>3 \mathrm{~cm}$ ) [14] and categorized as Child-Pugh A or B. Each of the hepatectomy and RFA groups included 115 patients. Although the tumor size was larger in patients in the hepatectomy group, the prognosis was significantly better in this group than that in the RFA group (5-year survival rate: $76 \%$ versus $55 \%$ ). Further analyses by subgroup in patients with solitary HCC measuring $3 \mathrm{~cm}$ or less, solitary HCC measuring 3 to $5 \mathrm{~cm}$, multinodular HCCs, or HCC patients with severe liver cirrhosis showed significantly better survival in the patients undergoing hepatectomy in all subgroups [15].

For more advanced HCCs, such as huge tumors measuring more than $10 \mathrm{~cm}$ in diameter, tumors associated with macroscopic vascular invasion, or tumors with extrahepatic metastases, surgical resection is advocated, because no other treatments such as local ablation, systemic chemotherapy, or liver transplantation are effective. Portal vein embolization (PVE), which was originally proposed as a strategy to decrease the risk of hepatic failure after extended hepatectomy for hilar cholangiocarcinoma [16], also has a role in increasing the patient suitability for hepatic resection in patients with HCC [17]. Although the regenerative capacity of the cirrhotic liver may be poor, the combination of PVE with TACE $[18,19]$, further combined with hepatic vein embolization, may help in increasing the patient suitability for hepatectomy [20]. Although studies encouraging aggressive surgery for advanced HCCs, as described below, were all retrospective in nature and showed good results in selected patients, surgery should be advocated if the safety is guaranteed by a low mortality and morbidity, because no other treatment has been demonstrated to be potentially as effective.

2.1. Large HCCs. Several studies have reported the shortand long-term outcomes of resection of extremely large ( $>10 \mathrm{~cm}$ in diameter) HCCs [21-24]. In these studies, 40 to 166 patients with HCC tumors measuring $10 \mathrm{~cm}$ or more in diameter were evaluated. Although relatively extensive hepatic resection was required, associated with an increased blood loss, the postoperative morbidity and mortality were comparable with those of hepatectomy for smaller HCCs. The mortality and 5-year overall survival rates were 2$3.3 \%$ and $28-33 \%$, respectively. Multiple tumors, vascular invasion, and impaired liver function were found to be predictors of poor survival. The safety and survival benefits shown in selected patients in these studies may justify aggressive resection for large HCCs.

Large HCCs should be treated by surgical resection as long as the liver function is maintained within a satisfactory range. Recently, nonalcoholic fatty liver disease (NAFLD) has been increasingly identified as a cause of HCC. Paradis et al. [25] compared the pathologic features of HCCs associated with metabolic syndrome or arising in a background of cryptogenic cirrhosis with those arising in a background of chronic liver disease. The former are larger in size, more frequently well differentiated and the background liver is less fibrotic. Therefore, the opportunity to resect indications for resection in patients with large HCCs is expected to increase.

2.2. HCCs with Macroscopic Vascular Invasion. Presence/ absence of macroscopic vascular invasion is one of the strongest predictors of the prognosis in patients with HCC. HCCs tend to invade adjacent venous tributaries, which is associated with an increased risk of intrahepatic or extrahepatic metastases [26-28]. Resection of HCCs with macroscopic vascular invasion or tumor thrombi is technically challenging and has been considered to yield limited survival benefit. However, the prognosis of HCCs with vascular invasion is even more dismal if they are left untreated. The median survival of untreated HCC patients with portal venous invasion is only 2.7 months [29]. Surgical resection leads to better survival outcomes than nonsurgical treatment strategies [30]. Several studies have shown poor survival 
benefit of surgical resection in HCC patients with portal venous invasion of the main trunk ( $\mathrm{Vp} 4$ [9]) or of a firstorder branch (right or left main trunk, Vp3) as compared with that in HCC patients with portal venous invasion of the secondary tributaries (Vp2) or further peripheral tributaries (Vp1) of the portal vein, and resection for HCCs with $\mathrm{Vp} 3$ or $\mathrm{Vp} 4$ is not recommended [31,32]. On the other hand, some authors have advocated aggressive resection even for HCCs with $\mathrm{Vp} 3$ or $\mathrm{Vp} 4$, reporting 5-year survival rates ranging from $10.9 \%$ to $42 \%$ [33-38]. Minagawa et al. previously reported the outcomes of 18 selected HCC patients with Vp3 or Vp4 treated by preoperative TACE followed by anatomical hepatic resection and reported a 5-year survival rate of $42 \%$ [34]. Based on the results of multivariate analyses, they proposed selection criteria for TACE followed by resection as no more than two nodules, a nonoccluded portal vein trunk, and an ICG-R15 of less than $20 \%$. As for the techniques for resection of a portal vein tumor thrombus, two types of procedures have been reported. One is resection of the involved segment of the portal vein; Wu et al. insisted on the surgical margin being secured by this technique [33]. The other is the peeling-off technique, in which a portal venotomy is placed and the tumor thrombus is detached and removed from the internal wall of the portal vein. Inoue et al. showed comparable postoperative overall and recurrencefree survivals between this peeling-off technique and the en bloc portal vein resection (5-year overall survival: $39 \%$ versus $41 \%, P=.90$; 5 -year recurrence-free survival: $23 \%$ versus $18 \%, P=.89$ ) [37].

As compared to portal venous invasion, HCC invasion of the bile duct or inferior vena cava is quite rare. In regard to the prognostic influence of bile duct tumor thrombi, Satoh et al. previously reported that there was no significant difference in the postoperative prognosis between patients with and without bile duct thrombi [39]. However, this result seemed to be influenced by the small number of patients with bile duct thrombi. More recently, Noda et al. reported the results of surgical resection of 22 patients with biliary tumor thrombi; they reported a 3 -year survival rate of $30 \%$, which was significantly worse than that of patients without biliary tumor thrombi. In the present study, portal or hepatic vein tumor thrombi, seen in 13 patients (59\%), was the only significant predictor of the prognosis [40]. Ikenaga et al. showed that bile duct invasion was associated with a worse prognosis irrespective of the degree of invasion, that is, patients with biliary invasion only to a third-order or more peripheral branch showed a similarly poor prognosis to those with more proximal bile duct invasion (first- or second-order branches or the common hepatic duct) [41]. Most of these studies advocated aggressive hepatic resection in selected HCC patients with macroscopic vasculobiliary invasion; however, additional preoperative or adjuvant treatments should be considered to improve the long-term outcomes.

2.3. Resection of Extrahepatic Metastases. Poon et al. reported limited benefit of aggressive resection of extrahepatic metastases when the extrahepatic lesion was solitary and/or the intrahepatic recurrence was well controlled [42]. Yang et al. reported that extrahepatic recurrence occurring after repetitive treatment for intrahepatic recurrence was associated with a better prognosis than simultaneous intrahepatic and extrahepatic recurrence or extrahepatic recurrence preceding intrahepatic recurrence; however, the 5-year survival rate after initial hepatic resection in 20 patients with the former type of extrahepatic recurrence was only $30 \%$ [43].

The lungs are the most common site of extrahepatic metastasis from HCC, with pulmonary metastases accounting for $50-60 \%$ of all extrahepatic metastases $[44,45]$. The outcomes of surgical resection of lung metastases have been increasingly reported recently, with the reported 5-year survival rate after pulmonary resection being in the range of $27-33 \%$; however, the number of patients included in each of these studies was small (8-61 patients), because, in most cases of pulmonary metastases, the lesions are multiple and surgical resection is contraindicated [46-49]. Kawamura et al. suggested that surgical resection may be considered in patients with up to three pulmonary lesions [49]. Kuo et al. showed that the disease-free interval and number of lesions were associated with the prognosis [47].

Surgical resection of other sites than the lung is reported only sporadically. Recently, Chan et al. reported the outcomes of surgical resection of extrahepatic metastases including in the lung, bone, brain, soft tissues, and heart and showed that resection of lung metastases was associated with the most favorable prognosis. Although the outcomes of resection of bone or brain metastases were dismal, with a 3-year survival rate of $9 \%$ and $0 \%$, respectively, even in these groups, patients who underwent surgical resection showed better survivals than patients who did not undergo surgical resection [50]. Sakamoto et al. reported four cases of resection of adrenal metastases. A literature review of 79 cases of adrenal metastases did not reveal any significant survival benefit of surgical resection. However, considering the risk of tumor rupture and venous invasion resulting in pulmonary embolism, or the technical difficulty of TACE due to the presence of three arteries feeding the adrenal grand, adrenalectomy may be a valid strategy, because it can be performed safely with minimal mortality [51]. Until other treatment alternatives are established to improve the patients' quality of life and life expectancy, surgical resection may be justified for the treatment of extrahepatic metastases.

\section{Anatomic Resection}

As described above, HCCs show a high predilection for invading the adjacent portal vein and, consequently, intrahepatic metastasis. On the other hand, extensive hepatic resection is contraindicated in most cases due to the severity of the background liver disease. To overcome this problem, Makuuchi et al. proposed anatomic subsegmentectomy [52]. In this type of hepatectomy, intraoperative ultrasonography is performed to identify the locations of the hepatic tumors. Dye (indigo carmine) is injected into the portal venous tributaries supplying the region containing the tumor. The area showing positive staining is then marked with electrocautery, and a parenchymal transection is performed. 
According to the location or size of the tumor and the background liver function, a part of one Couinaud's segment or more than one segment is resected. The influence of anatomic resection on the postoperative prognosis has been evaluated recently. Most studies have shown the superiority of anatomic resection for prolonged overall or recurrencefree survival, especially in selected patients with solitary HCCs, small HCCs, or HCCs fulfilling the Milan criteria [14, 53-57]. Among the studies, the largest series was a Japanese nationwide survey including 5781 patients who underwent anatomic subsegmentectomy or nonanatomic minor hepatectomy for solitary HCCs. The overall survival was marginally better in the anatomic resection group in the overall analysis $(P=.053)$. Subgroup analysis showed significantly better disease-free survival following anatomic resection in patients with $\mathrm{HCCs}$ measuring 2 to $5 \mathrm{~cm}$ in diameter $(P=.0005)$ [56].

\section{Resection of Recurrent HCCs}

The most common site of recurrence is the remnant liver, which accounts for $85 \%$ to $90 \%$ of the initial recurrences [58]. The reported incidence of intrahepatic recurrence within 2 years after primary hepatic resection is $70 \%$ [58]. The effectiveness of systemic chemotherapy for HCCs has not yet been established; therefore, in the absence of extrahepatic metastases, local treatment is currently the only approach that can yield long-term survival in patients with recurrent HCC. Several studies have demonstrated that repeat hepatectomy yields a better prognosis than other nonsurgical treatments, with a 5-year survival rate after repeat hepatectomy of around 50-70\%, which is almost comparable to the survival rate after first hepatectomy [59-63]. Wu et al. reviewed the outcomes of patients who underwent hepatectomy for HCC up to four times and showed that patients undergoing their second and third hepatectomies, but not those undergoing the fourth hepatectomy, showed significantly better survivals than those who did not undergo repeat hepatectomy [64]. All of these studies, however, were retrospective in nature, and the selection bias for patients who underwent repeat hepatic resection must be considered. Repeat hepatic resection is indicated for only a limited proportion of patients, because multiple intrahepatic tumor recurrences and/or impaired liver function due mostly to the background liver diseases represent contraindications to repeat hepatectomy. Minagawa et al. analyzed the prognostic predictors in patients undergoing repeat hepatectomy and reported a solitary HCC at primary hepatectomy, diseasefree interval of 1 year or more, and absence of portal venous invasion at the second hepatectomy as independent predictors of a favorable prognosis [65]. Repeating locoregional treatment such as ethanol injection (PEI), radiofrequency ablation (RFA), or TACE, for an intrahepatic recurrence may also help in prolonging patient survival [66-70]. Locoregional treatments may be repeated as long as there are no extrahepatic recurrences and the remnant liver function is reasonably adequate. Surgical resection after PEI/RFA can also be considered for recurrent HCC [71].

\section{Preoperative and Adjuvant Treatments}

Unlike the case for colorectal liver metastases, in which preoperative chemotherapy may improve the prognosis of patients with either resectable or initially unresectable tumors [72-75], there is no established preoperative adjuvant strategy to improve the prognosis in HCC patients. Previous studies have evaluated the role of preoperative TACE, but the long-term outcomes remain controversial. Most studies, including one RCT, have shown no survival benefit [76-82]. Furthermore, some studies identified preoperative TACE as a predictor of extrahepatic metastasis $[83,84]$ or worse survival $[79,85]$. On the other hand, selected patients with TACE-mediated complete necrosis showed a significantly more favorable prognosis after hepatic resection [77, 83, 85-88]. These results may be explained by Adachi's hypothesis that viable cancer cells are less firmly attached and are more likely to disseminate into the blood stream during surgical manipulation following incomplete necrosis by TACE [86]. Considering the low incidence of complete necrosis achieved by TACE, ranging from $6 \%$ to $29 \%$ according to previous reports [77, 87, 88], routine implementation of preoperative TACE may not be recommended. Several other studies report that preoperative TACE may improve the disease-free and overall survivals in selected cases, such as HCC patients with macroscopic portal venous invasion [34], advanced-stage tumors [89], severe liver dysfunction (ICG-R15 $\geqq 17 \%$ ) [90], or centrally located large tumors [84]. One study showed that TACE converted initially unresectable HCCs to resectable tumors, yielding a 5-year survival rate of 56\% [91]. Taking all the above reports into consideration, TACE as a postoperative adjuvant treatment seems to have a role in improving the prognosis of patients, especially of those having advanced HCCs with portal venous invasion or intrahepatic metastases $[92,93]$.

A multitargeted agent sorafenib, which exerts both antiangiogenic and antiproliferative effects, is the first systemic agent to have yielded survival benefit in patients with advanced HCCs in a phase III, randomized, placebocontrolled trial [94]. However, the benefit is limited (median overall survival: 10.7 months versus 7.9 months) and treatment with this drug has not yet been validated as a preoperative or adjuvant treatment. Until date, other modalities such as hepatic artery infusion of radionuclide Yttrium-90 microspheres, or combined subcutaneous interferon alpha and intraarterial infusion chemotherapy, seem to be more promising strategies than sorafenib treatment to downstage advanced HCCs, including cases with macroscopic portal venous invasion [95-100]. Especially, two recent reports from Japanese groups showed a response rate of $33-52 \%$ following combined interferon and intraarterial 5-fluorouracil therapy in advanced HCC patients with portal venous invasion. These results suggest further increase in the number of candidates suitable for surgical resection among patients with initially unresectable HCCs. 


\section{Laparoscopic Hepatectomy}

Laparoscopic liver resection was first reported in the early 1990s for partial resection of segment 6 for a $6 \mathrm{~cm}$ focal nodular hyperplasia and wedge resection of segment 5 for colorectal liver metastases [101]. Since then, the number of reported cases of laparoscopic liver resection has increased dramatically, especially over the last 5 years [102]. Although some surgeons are still skeptical about the oncological curative potential of laparoscopic surgery and evaluation of the long-term prognosis is required to justify the minimally invasive approaches for hepatic malignancies, several specialized centers have expanded the indications of laparoscopic hepatectomy from benign tumors to malignant tumors, including HCCs [103-105] and from wedge resection of the anterolateral segments to major hepatectomy or resection of the posterosuperior segments, such as segment 8,7 , or $1[106,107]$. The consensus conference by 45 experts held in October 2008 proposed that the most suitable candidates among HCC patients for laparoscopic hepatectomy are, in general, those with solitary lesions measuring $5 \mathrm{~cm}$ or less in diameter, located in the peripheral segments. The conference also proposed that major hepatectomy or other technically complicated procedures should be left to experienced surgeons [108].

Several surgeons advocate laparoscopic resection for HCC, especially that in a cirrhotic liver, due to its lessinvasive characteristic, because less liver mobilization is required and the amount of intravenous fluid needed is reduced due to the minimized insensible fluid loss occurring during this operation as compared with that during open liver resection. Fluid accumulation in the third space decreases, which may be expected to be associated with a reduced risk of prolonged postoperative accumulation of ascites [109-112]. On the other hand, higher morbidity and mortality have been reported, even from highly specialized institutions [113]. As for the survival outcomes, only a few reports based on studies of a small number of cases each have been published, which have shown a comparable short-term prognosis in patients undergoing laparoscopic hepatectomy as compared to that in patients undergoing open hepatectomy $[109,111,112]$; further investigation is required for validation. A significant learning curve is inevitable to establish the safety and feasibility of laparoscopic surgery [114] and this is one of the major limitations in relation to the popularization of laparoscopic hepatectomy. The indications for laparoscopic resection should be appropriately decided according to the types and conditions of the tumors and the technical skills of the surgeons. More importantly, the safety and oncological curative potential must be accorded priority; therefore, conversion to an open procedure should be expedited if bleeding cannot be controlled laparoscopically, or an adequate resection margin cannot be obtained, or adhesions preclude the laparoscopic procedure [102, 113].

\section{Liver Transplantation for HCCs}

The Milan criteria, which were published in 1996 [14], have served as appropriate selection criteria for patients with HCC who are potential candidates for liver transplantation. The criteria have been validated by numerous subsequent studies, with patients fulfilling the Milan criteria showing significantly better survivals than those not fulfilling the criteria, in both cadaveric and living donor liver transplantation (3year survival rate of $79-91 \%$ versus $60-66 \%$ ) $[115,116]$. The criteria are also utilized by the United Network for Organ Sharing [117]. However, the Milan criteria are rather restrictive, permitting liver transplantation in only a limited proportion of patients with HCC, and therefore, expansion of the criteria has been proposed. The most representative example of such expanded criteria is the University of San Francisco (UCSF) criteria proposed by Yao et al., which include solitary tumor $\leqq 6.5 \mathrm{~cm}$, or two or three nodules with the largest lesion $\leqq 4.5 \mathrm{~cm}$ and total tumor diameter $\leqq$ $8 \mathrm{~cm}$ [118]. These criteria were also validated by subsequent studies from high-volume transplantation centers, which reported overall 5-year survival rates of 52-64\% [119, 120]. In cases of living donor liver transplantation, it may be possible to expand the selection criteria more readily, because the donors would be expected to have a stronger motivation for self-giving and dedication to the recipients. In The University of Tokyo, patients with HCC having up to 5 nodules with a maximal diameter of $5 \mathrm{~cm}$ (the $5-5$ rule) have been treated by transplantation, and the 5 -year survival rate in a series of 72 patients treated according to this 5-5 rule was reported to be $75 \%$ [121]. Other Japanese institutions have reported a more aggressive approach to living donor liver transplantation, operating on HCC patients with no extrahepatic metastases or macroscopic vascular invasion, but regardless of the size and number of tumors [122-124]. Soejima et al. have insisted that patients with small multiple HCCs should be included as candidates for LT [122]. In their report of $60 \mathrm{HCC}$ patients who underwent living donor liver transplantation, 23 patients with 4 to 10 tumors and 12 patients with more than 10 tumors showed 3-year recurrence-free survival rates of $75 \%$ and $72 \%$, respectively, and only preoperative des-gamma-carboxy prothrombin (DCP) levels of $>300 \mathrm{mAU} / \mathrm{ml}$ and tumor size $>5 \mathrm{~cm}$ were identified as independent prognostic factors. They validated this result recently in an extended series of 90 patients. In this study, a 5-year survival rate of $83 \%$ was seen in 85 HCC patients meeting the criteria of a maximal tumor size of less than $5 \mathrm{~cm}$ or DCP level of less than $300 \mathrm{mAU} / \mathrm{ml}$ [124]. Similarly, Ito et al. analyzed the prognostic factors in their series of 125 patients and proposed the following criteria: 10 or fewer tumors, with each tumor measuring $5 \mathrm{~cm}$ or less in diameter, and a DCP level of $400 \mathrm{mAU} / \mathrm{ml}$ or less. The 5 -year survival rate in 78 patients meeting these criteria was $87 \%$ [123].

In most Western countries as in Japan, hepatitis $C$ virus (HCV) is the most common etiological agent associated with HCC. Hepatitis C recurs inevitably in the transplanted liver, and progression to graft cirrhosis occurs more rapidly under immunsuppression [125], which becomes another possible cause of the patients' death in addition to tumor recurrence. Therefore, a special postoperative immunosuppression protocol, such as a steroid-free protocol [126, 127], or cyclosporine-based instead of a tacrolimus-based protocol 
should be considered [128]. Preemptive antiviral therapy using interferon and ribavirin may also help in improving the survival of the recipients, by reducing the risk of progression of fibrosis in the grafts $[129,130]$.

Under the current circumstance of shortage of donors, opportunities for liver transplantation are limited. Therefore, nontransplant treatments, including open or laparoscopic hepatic resection, PEI/RFA, or TACE, should be the treatment modalities of first choice in HCC patients with compensated liver cirrhosis. Even in patients with decompensated cirrhosis, these modalities may well be used as a bridge to transplantation. Several studies have shown that secondary or salvage transplantation for downstaged HCC by nontransplant treatment or for recurrent HCC after initial treatment results in acceptable survival outcomes, especially if the HCC meets the Milan criteria prior to transplantation [131-134]. The reported 5-year survival rates after secondary transplantation from these studies are in the range of $60-80 \%$. Interestingly, Takada et al. reported that patients with one or two non-transplant treatments prior to liver transplantation showed better outcomes after transplantation than patients without any previous treatment and also patients with 3 or more treatments. They concluded that patients developing recurrent HCCs should be referred for transplantation before further nontransplant treatment is repeated [135]. Further investigation is required to determine the appropriate timing of transplantation.

\section{Conclusions}

The indications for surgical resection in patients with HCC have expanded concomitantly with the establishment of the safety and feasibility of aggressive resection. However, a high incidence of postoperative recurrence is still an obstacle to the achievement of long-term survival in advanced HCC patients. A multimodality approach is required, especially for patients with recurrent HCC or HCC with background liver cirrhosis, which preclude hepatic resection. The benefit of systemic chemotherapy for extrahepatic metastases is still limited, and the development of better pharmacological agents in the future is expected. Most studies referred to in this paper were retrospective in nature. Although RCTs may not be ethically appropriate in all clinical researches, more prospective studies are needed to accumulate evidence for the establishment of multidisciplinary treatments for HCC.

\section{References}

[1] J. M. Llovet, J. Fuster, and J. Bruix, "Intention-to-treat analysis of surgical treatment for early hepatocellular carcinoma: resection versus transplantation," Hepatology, vol. 30 , no. 6, pp. 1434-1440, 1999.

[2] J. Bruix, M. Sherman, J. M. Llovet et al., "Clinical management of hepatocellular carcinoma. Conclusions of the Barcelona-2000 EASL conference. European association for the study of the liver," Journal of Hepatology, vol. 35, no. 3, pp. 421-430, 2001.

[3] J. M. Llovet, C. Brú, and J. Bruix, "Prognosis of hepatocellular carcinoma: the BCLC staging classification," Seminars in Liver Disease, vol. 19, no. 3, pp. 329-337, 1999.
[4] J. Bruix and M. Sherman, "Management of hepatocellular carcinoma," Hepatology, vol. 42, no. 5, pp. 1208-1236, 2005.

[5] T. Ishizawa, K. Hasegawa, T. Aoki et al., "Neither multiple tumors nor portal hypertension are surgical contraindications for hepatocellular carcinoma," Gastroenterology, vol. 134, no. 7, pp. 1908-1916, 2008.

[6] L. Capussotti, A. Ferrero, L. Viganò, R. Polastri, and M. Tabone, "Liver resection for HCC with cirrhosis: surgical perspectives out of EASL/AASLD guidelines," European Journal of Surgical Oncology, vol. 35, no. 1, pp. 11-15, 2009.

[7] S. T. Fan, C. M. Lo, C. L. Liu et al., "Hepatectomy for hepatocellular carcinoma: toward zero hospital deaths," The Annals of Surgery, vol. 229, no. 3, pp. 322-330, 1999.

[8] H. Imamura, Y. Seyama, N. Kokudo et al., "One thousand fifty-six hepatectomies without mortality in 8 years," Archives of Surgery, vol. 138, no. 11, pp. 1198-1206, 2003.

[9] Japan LCSGo, General Rules for the Clinical and Pathological Study of Primary Liver Cancer, Kenehara, Tokyo, Japan, 2003.

[10] M. Makuuchi, N. Kokudo, S. Arii et al., "Development of evidence-based clinical guidelines for the diagnosis and treatment of hepatocellular carcinoma in Japan," Hepatology Research, vol. 38, no. 1, pp. 37-51, 2008.

[11] G. T. Huang, P. O. H. Lee, Y. M. Tsang et al., "Percutaneous ethanol injection versus surgical resection for the treatment of small hepatocellular carcinoma: a prospective study," The Annals of Surgery, vol. 242, no. 1, pp. 36-42, 2005.

[12] M. S. Chen, J. Q. Li, Y. Zheng et al., "A prospective randomized trial comparing percutaneous local ablative therapy and partial hepatectomy for small hepatocellular carcinoma," The Annals of Surgery, vol. 243, no. 3, pp. 321328, 2006.

[13] K. Hasegawa, N. Kokudo, and M. Makuuchi, "Surgery or ablation for hepatocellular carcinoma?" The Annals of surgery, vol. 247, no. 3, pp. 557-558, 2008.

[14] V. Mazzaferro, E. Regalia, R. Doci et al., "Liver transplantation for the treatment of small hepatocellular carcinomas in patients with cirrhosis," The New England Journal of Medicine, vol. 334, no. 11, pp. 693-699, 1996.

[15] J. Huang, L. Yan, Z. Cheng et al., "A randomized trial comparing radiofrequency ablation and surgical resection for HCC conforming to the Milan criteria," The Annals of Surgery, vol. 252, no. 6, pp. 903-912, 2010.

[16] M. Makuuchi, B. L. Thai, K. Takayasu et al., "Preoperative portal embolization to increase safety of major hepatectomy for hilar bile duct carcinoma: a preliminary report," Surgery, vol. 107, no. 5, pp. 521-527, 1990.

[17] M. Palavecino, Y. S. Chun, D. C. Madoff et al., "Major hepatic resection for hepatocellular carcinoma with or without portal vein embolization: perioperative outcome and survival," Surgery, vol. 145, no. 4, pp. 399-405, 2009.

[18] T. Aoki, H. Imamura, K. Hasegawa et al., "Sequential preoperative arterial and portal venous embolizations in patients with hepatocellular carcinoma," Archives of Surgery, vol. 139, no. 7, pp. 766-774, 2004.

[19] S. Ogata, J. Belghiti, O. Farges, D. Varma, A. Sibert, and V. Vilgrain, "Sequential arterial and portal vein embolizations before right hepatectomy in patients with cirrhosis and hepatocellular carcinoma," British Journal of Surgery, vol. 93, no. 9, pp. 1091-1098, 2006.

[20] S. Hwang, S. G. Lee, G. I. Y. Ko et al., "Sequential preoperative ipsilateral hepatic vein embolization after portal vein 
embolization to induce further liver regeneration in patients with hepatobiliary malignancy," The Annals of Surgery, vol. 249, no. 4, pp. 608-616, 2009.

[21] N. H. Lee, G. Y. Chau, W. Y. Lui, K. L. King, S. H. Tsay, and C. W. Wu, "Surgical treatment and outcome in patients with a hepatocellular carcinoma greater than $10 \mathrm{~cm}$ in diameter," British Journal of Surgery, vol. 85, no. 12, pp. 1654-1657, 1998.

[22] R. T. P. Poon, S. T. Fan, and J. Wong, "Selection criteria for hepatic resection in patients with large hepatocellular carcinoma larger than $10 \mathrm{~cm}$ in diameter," The Journal of the American College of Surgeons, vol. 194, no. 5, pp. 592-602, 2002.

[23] K. H. Liau, L. Ruo, J. Shia et al., "Outcome of partial hepatectomy for large (>10 cm) hepatocellular carcinoma," Cancer, vol. 104, no. 9, pp. 1948-1955, 2005.

[24] D. Pandey, K. H. Lee, C. T. Wai, G. Wagholikar, and K. C. Tan, "Long term outcome and prognostic factors for large hepatocellular carcinoma ( $10 \mathrm{~cm}$ or more) after surgical resection," Annals of Surgical Oncology, vol. 14, no. 10, pp. 2817-2823, 2007.

[25] V. Paradis, S. Zalinski, E. Chelbi et al., "Hepatocellular carcinomas in patients with metabolic syndrome often develop without significant liver fibrosis: a pathological analysis," The Journal of Hepatology, vol. 49, no. 3, pp. 851-859, 2009.

[26] "Predictive factors for long term prognosis after partial hepatectomy for patients with hepatocellular carcinoma in Japan. The Liver cancer study group of Japan," Cancer, vol. 74, no. 10, pp. 2772-2780, 1994.

[27] J. N. Vauthey, D. Klimstra, D. Franceschi, Y. Tao, J. Fortner, and L. Blumgart, "Factors affecting long-term outcome after hepatic resection for hepatocellular carcinoma," The American Journal of Surgical Pathology, vol. 169, no. 1, pp. 28-34, 1995.

[28] M. Kanda, R. Tateishi, H. Yoshida et al., "Extrahepatic metastasis of hepatocellular carcinoma: incidence and risk factors," Liver International, vol. 28, no. 9, pp. 1256-1263, 2008.

[29] J. M. Llovet, J. Bustamante, A. Castells et al., "Natural history of untreated nonsurgical hepatocellular carcinoma: rationale for the design and evaluation of therapeutic trials," Hepatology, vol. 29, no. 1, pp. 62-67, 1999.

[30] T. M. Pawlik, R. T. Poon, E. K. Abdalla et al., "Hepatectomy for hepatocellular carcinoma with major portal or hepatic vein invasion: results of a multicenter study," Surgery, vol. 137, no. 4, pp. 403-410, 2005.

[31] J. Shi, E. C. H. Lai, N. Li et al., "Surgical treatment of hepatocellular carcinoma with portal vein tumor thrombus," Annals of Surgical Oncology, pp. 1-8, 2010.

[32] D. X. Lin, Q. Y. Zhang, X. Li, Q. W. Ye, F. Lin, and L. L. $\mathrm{Li}$, "An aggressive approach leads to improved survival in hepatocellular carcinoma patients with portal vein tumor thrombus," Journal of Cancer Research and Clinical Oncology, pp. 1-11, 2010.

[33] C. C. Wu, S. R. Hsieh, J. T. Chen et al., "An appraisal of liver and portal vein resection for hepatocellular carcinoma with tumor thrombi extending to portal bifurcation," Archives of Surgery, vol. 135, no. 11, pp. 1273-1279, 2000.

[34] M. Minagawa, M. Makuuchi, T. Takayama, and K. Ohtomo, "Selection criteria for hepatectomy in patients with hepatocellular carcinoma and portal vein tumor thrombus," The Annals of Surgery, vol. 233, no. 3, pp. 379-384, 2001.
[35] I. Ikai, E. Hatano, S. Hasegawa et al., "Prognostic index for patients with hepatocellular carcinoma combined with tumor thrombosis in the major portal vein," The Journal of the American College of Surgeons, vol. 202, no. 3, pp. 431-438, 2006.

[36] Y. P. Le Treut, J. Hardwigsen, P. Ananian et al., "Resection of hepatocellular carcinoma with tumor thrombus in the major vasculature. a European case-control series," The Journal of Gastrointestinal Surgery, vol. 10, no. 6, pp. 855-862, 2006.

[37] Y. Inoue, K. Hasegawa, T. Ishizawa et al., "Is there any difference in survival according to the portal tumor thrombectomy method in patients with hepatocellular carcinoma?" Surgery, vol. 145, no. 1, pp. 9-19, 2009.

[38] D. Ban, K. Shimada, Y. Yamamoto et al., "Efficacy of a hepatectomy and a tumor thrombectomy for hepatocellular carcinoma with tumor thrombus extending to the main portal vein," The Journal of Gastrointestinal Surgery, vol. 13, no. 11, pp. 1921-1928, 2009.

[39] S. Satoh, I. Ikai, G. Honda et al., "Clinicopathologic evaluation of hepatocellular carcinoma with bile duct thrombi," Surgery, vol. 128, no. 5, pp. 779-783, 2000.

[40] T. Noda, H. Nagano, Y. Tomimaru et al., "Prognosis of hepatocellular carcinoma with biliary tumor thrombi after liver surgery," Surgery, vol. 149, no. 3, pp. 371-377, 2011.

[41] N. Ikenaga, K. Chijiiwa, K. Otani, J. Ohuchida, S. Uchiyama, and K. Kondo, "Clinicopathologic characteristics of hepatocellular carcinoma with bile duct invasion," The Journal of Gastrointestinal Surgery, vol. 13, no. 3, pp. 492-497, 2009.

[42] R. T. P. Poon, S. T. Fan, C. B. O’Suilleabhain, and J. Wong, "Aggressive management of patients with extrahepatic and intrahepatic recurrences of hepatocellular carcinoma by combined resection and locoregional therapy," The Journal of the American College of Surgeons, vol. 195, no. 3, pp. 311-318, 2002.

[43] Y. Yang, H. Nagano, and H. Ota, "Patterns and clinicopathologic features of extrahepatic recurrence of hepatocellular carcinoma after curative resection," Surgery, vol. 141, no. 2, pp. 196-202, 2007.

[44] S. Katyal, J. H. Oliver III, M. S. Peterson, J. V. Ferris, B. S. Carr, and R. L. Baron, "Extrahepatic metastases of hepatocellular carcinoma," Radiology, vol. 216, no. 3, pp. 698-703, 2000.

[45] M. Natsuizaka, T. Omura, T. Akaike et al., "Clinical features of hepatocellular carcinoma with extrahepatic metastases," The Journal of Gastroenterology and Hepatology, vol. 20, no. 11, pp. 1781-1787, 2005.

[46] Y. Tomimaru, Y. O. Sasaki, T. Yamada et al., "The significance of surgical resection for pulmonary metastasis from hepatocellular carcinoma," The American Journal of Surgery, vol. 192, no. 1, pp. 46-51, 2006.

[47] S. W. Kuo, Y. L. Chang, P. M. Huang et al., "Prognostic factors for pulmonary metastasectomy in hepatocellular carcinoma," Annals of Surgical Oncology, vol. 14, no. 2, pp. 992-997, 2007.

[48] K. Norimasa, K. Haruhiko, S. Kenji, A. Hisao, S. Kazuaki, and T. Ryosuke, "Surgical treatment of pulmonary metastasis from hepatocellular carcinoma," Hepato-Gastroenterology, vol. 54, no. 73, pp. 152-156, 2007.

[49] M. Kawamura, J. Nakajima, H. Matsuguma et al., "Surgical outcomes for pulmonary metastases from hepatocellular carcinoma," European Journal of Cardio-Thoracic Surgery, vol. 34, no. 1, pp. 196-199, 2008.

[50] K. M. Chan, M. C. Yu, T. J. Wu et al., "Efficacy of surgical resection in management of isolated extrahepatic metastases of hepatocellular carcinoma," World Journal of Gastroenterology, vol. 15, no. 43, pp. 5481-5488, 2009. 
[51] Y. Sakamoto, K. Kubota, M. Mori et al., "Surgical management for adrenal gland metastasis of hepatocellular carcinoma," Hepato-Gastroenterology, vol. 46, no. 26, pp. 1036-1041, 1999.

[52] M. Makuuchi, H. Hasegawa, and S. Yamazaki, "Ultrasonically guided subsegmentectomy," Surgery Gynecology \& Obstetrics, vol. 161, no. 4, pp. 346-350, 1985.

[53] H. Imamura, Y. Matsuyama, Y. Miyagawa et al., "Prognostic significance of anatomical resection and des- $\gamma$-carboxy prothrombin in patients with hepatocellular carcinoma," The British Journal of Surgery, vol. 86, no. 8, pp. 1032-1038, 1999.

[54] M. Yamamoto, K. Takasaki, T. Ohtsubo, H. Katsuragawa, C. Fukuda, and S. Katagiri, "Effectiveness of systematized hepatectomy with Glisson's pedicle transection at the hepatic hilus for small nodular hepatocellular carcinoma: retrospective analysis," Surgery, vol. 130, no. 3, pp. 443-448, 2001.

[55] K. Hasegawa, N. Kokudo, H. Imamura et al., "Prognostic impact of anatomic resection for hepatocellular carcinoma," The Annals of Surgery, vol. 242, no. 2, pp. 252-259, 2005.

[56] S. Eguchi, T. Kanematsu, S. Arii et al., "Comparison of the outcomes between an anatomical subsegmentectomy and a non-anatomical minor hepatectomy for single hepatocellular carcinomas based on a Japanese nationwide survey," Surgery, vol. 143, no. 4, pp. 469-475, 2008.

[57] T. Kamiyama, K. Nakanishi, H. Yokoo, H. Kamachi, M. Matsushita, and S. Todo, "The impact of anatomical resection for hepatocellular carcinoma that meets the Milan criteria," The Journal of Surgical Oncology, vol. 101, no. 1, pp. 54-60, 2010.

[58] S. Arii, K. Teramoto, T. Kawamura et al., "Characteristics of recurrent hepatocellular carcinoma in Japan and our surgical experience," The Journal of Hepato-Biliary-Pancreatic Surgery, vol. 8, no. 5, pp. 397-403, 2001.

[59] M. Shimada, K. Takenaka, T. Gion et al., "Prognosis of recurrent hepatocellular carcinoma: a 10-year surgical experience in Japan," Gastroenterology, vol. 111, no. 3, pp. 720-726, 1996.

[60] R. T. P. Poon, S. T. Fan, C. M. Lo, C. L. Liu, and J. Wong, "Intrahepatic recurrence after curative resection of hepatocellular carcinoma: long-term results of treatment and prognostic factors," The Annals of Surgery, vol. 229, no. 2, pp. 216-222, 1999.

[61] Y. Nakajima, S. Ko, T. Kanamura et al., "Repeat liver resection for hepatocellular carcinoma," The Journal of the American College of Surgeons, vol. 192, no. 3, pp. 339-344, 2001.

[62] K. Sugimachi, S. Maehara, S. Tanaka, M. Shimada, and K. Sugimachi, "Repeat hepatectomy is the most useful treatment for recurrent hepatocellular carcinoma," The Journal of Hepato-Biliary-Pancreatic Surgery, vol. 8, no. 5, pp. 410-416, 2001.

[63] T. Itamoto, H. Nakahara, H. Amano et al., "Repeat hepatectomy for recurrent hepatocellular carcinoma," Surgery, vol. 141, no. 5, pp. 589-597, 2007.

[64] C. C. Wu, S. B. Cheng, D. C. Yeh, J. Wang, and F. K. P'eng, "Second and third hepatectomies for recurrent hepatocellular carcinoma are justified," British Journal of Surgery, vol. 96, no. 9, pp. 1049-1057, 2009.

[65] M. Minagawa, M. Makuuchi, T. Takayama, and N. Kokudo, "Selection criteria for repeat hepatectomy in patients with recurrent hepatocellular carcinoma," The Annals of Surgery, vol. 238, no. 5, pp. 703-710, 2003.

[66] R. T. P. Poon, H. Ngan, C. M. Lo, C. L. Liu, S. T. Fan, and J. Wong, "Transarterial chemoembolization for inoperable hepatocellular carcinoma and postresection intrahepatic recurrence," Journal of Surgical Oncology, vol. 73, no. 2, pp. 109-114, 2000.

[67] W. Yang, M. H. Chen, and S. S. Yin, "Radiofrequency ablation of recurrent hepatocellular carcinoma after hepatectomy: therapeutic efficacy on early- and late-phase recurrence," AJR. American Journal of Roentgenology, vol. 185, supplement 5, pp. S275-S283, 2006.

[68] K. Taura, I. Ikai, E. Hatano, H. Fujii, N. Uyama, and Y. Shimahara, "Implication of frequent local ablation therapy for intrahepatic recurrence in prolonged survival of patients with hepatocellular carcinoma undergoing hepatic resection: an analysis of 610 patients over 16 years old," The Annals of Surgery, vol. 244, no. 2, pp. 265-273, 2006.

[69] D. Choi, H. K. Lim, H. Rhim et al., "Percutaneous radiofrequency ablation for recurrent hepatocellular carcinoma after hepatectomy: long-term results and prognostic factors," Annals of Surgical Oncology, vol. 14, no. 8, pp. 2319-2329, 2007.

[70] Y. Okuwaki, T. Nakazawa, S. Kokubu et al., "Repeat radiofrequency ablation provides survival benefit in patients with intrahepatic distant recurrence of hepatocellular carcinoma," The American Journal of Gastroenterology, vol. 104, no. 11, pp. 2747-2753, 2009.

[71] N. Portolani, G. L. Baiocchi, A. Coniglio et al., "Sequential multidisciplinary treatment of hepatocellular carcinoma: the role of surgery as rescue therapy for failure of percutaneous ablation therapies," The Journal of Surgical Oncology, vol. 100, no. 7, pp. 580-584, 2009.

[72] R. Adam, V. Delvart, G. Pascal et al., "Rescue surgery for unresectable colorectal liver metastases downstaged by chemotherapy: a model to predict long-term survival," The Annals of Surgery, vol. 240, no. 4, pp. 644-658, 2004.

[73] B. Nordlinger, H. Sorbye, B. Glimelius et al., "Perioperative chemotherapy with FOLFOX4 and surgery versus surgery alone for resectable liver metastases from colorectal cancer (EORTC Intergroup trial 40983): a randomised controlled trial," The Lancet, vol. 371, no. 9617, pp. 1007-1016, 2008.

[74] R. Adam, D. A. Wicherts, R. J. de Haas et al., "Complete pathologic response after preoperative chemotherapy for colorectal liver metastases: myth or reality?" The Journal of Clinical Oncology, vol. 26, no. 10, pp. 1635-1641, 2008.

[75] D. G. Blazer, Y. Kishi, D. M. Maru et al., "Pathologic response to preoperative chemotherapy: a new outcome end point after resection of hepatic colorectal metastases," The Journal of Clinical Oncology, vol. 26, no. 33, pp. 5344-5351, 2008.

[76] S. Yamasaki, H. Hasegawa, H. Kinoshita et al., "A prospective randomized trial of the preventive effect of pre-operative transcatheter arterial embolization against recurrence of hepatocellular carcinoma," Japanese Journal of Cancer Research, vol. 87, no. 2, pp. 206-211, 1996.

[77] T. Harada, K. Matsuo, T. Inoue, S. Tamesue, T. Inoue, and H. Nakamura, "Is preoperative hepatic arterial chemoembolization safe and effective for hepatocellular carcinoma?" The Annals of Surgery, vol. 224, no. 1, pp. 4-9, 1996.

[78] G. E. Gerunda, D. Neri, R. Merenda et al., "Role of transarterial chemoembolization before liver resection for hepatocarcinoma," Liver Transplantation, vol. 6, no. 5, pp. 619-626, 2000.

[79] A. Sasaki, Y. Iwashita, K. Shibata, M. Ohta, S. Kitano, and M. Mori, "Preoperative transcatheter arterial chemoembolization reduces long-term survival rate after hepatic resection for resectable hepatocellular carcinoma," European Journal of Surgical Oncology, vol. 37, no. 7, pp. 773-779, 2006. 
[80] G. I. H. Choi, D. H. Kim, C. M. Kang et al., "Is preoperative transarterial chemoembolization needed for a resectable hepatocellular carcinoma?" World Journal of Surgery, vol. 31, no. 12, pp. 2370-2377, 2007.

[81] W. P. Zhou, E. C. H. Lai, A. J. Li et al., "A prospective, randomized, controlled trial of preoperative transarterial chemoembolization for resectable large hepatocellular carcinoma," The Annals of Surgery, vol. 249, no. 2, pp. 195-202, 2009.

[82] K. T. Lee, Y. W. Lu, S. N. Wang et al., "The effect of preoperative transarterial chemoembolization of resectable hepatocellular carcinoma on clinical and economic outcomes," The Journal of Surgical Oncology, vol. 99, no. 6, pp. 343-350, 2009.

[83] C. C. Wu, Y. Z. Ho, W. L. Ho, T. C. Wu, T. J. Liu, and F. K. P'Eng, "Preoperative transcatheter arterial chemoembolization for resectable large hepatocellular carcinoma: a reappraisal," British Journal of Surgery, vol. 82, no. 1, pp. 122 126, 1995.

[84] X. P. Chen, D. Y. Hu, Z. W. Zhang et al., "Role of mesohepatectomy with or without transcatheter arterial chemoembolization for large centrally located hepatocellular carcinoma," Digestive Surgery, vol. 24, no. 3, pp. 208-213, 2007.

[85] I. S. Kim, Y. S. Lim, H. C. Lee, D. J. Suh, Y. J. Lee, and S. G. Lee, "Pre-operative transarterial chemoembolization for resectable hepatocellular carcinoma adversely affects postoperative patient outcome," Alimentary Pharmacology and Therapeutics, vol. 27, no. 4, pp. 338-345, 2008.

[86] E. Adachi, T. Matsumata, T. Nishizaki, H. Hashimoto, M. Tsuneyoshi, and K. Sugimachi, "Effects of preoperative transcatheter hepatic arterial chemoembolization for hepatocellular carcinoma: the relationship between postoperative course and tumor necrosis," Cancer, vol. 72, no. 12, pp. 3593 3598, 1993.

[87] O. Matsui, M. Kadoya, J. Yoshikawa et al., "Small hepatocellular carcinoma: treatment with subsegmental transcatheter arterial embolization," Radiology, vol. 188, no. 1, pp. 79-83, 1993.

[88] M. Uchida, H. Kohno, H. Kubota et al., "Role of preoperative transcatheter arterial oily chemoembolization for resectable hepatocellular carcinoma," World Journal of Surgery, vol. 20, no. 3, pp. 326-331, 1996.

[89] H. Sugo, S. Futagawa, T. Beppu, M. Fukasawa, and K. Kojima, "Role of preoperative transcatheter arterial chemoembolization for resectable hepatocellular carcinoma: relation between postoperative course and the pattern of tumor recurrence," World Journal of Surgery, vol. 27, no. 12, pp. 1295-1299, 2003.

[90] M. Kaibori, N. Tanigawa, Y. Matsui et al., "Influence of transcatheter arterial chemoembolization on the prognosis after hepatectomy for hepatocellular carcinoma in patients with severe liver dysfunction," Anticancer Research, vol. 26, no. 5, pp. 3685-3692, 2006.

[91] J. Fan, Z. Y. Tang, Y. Q. Yu et al., "Improved survival with resection after transcatheter arterial chemoembolization (TACE) for unresectable hepatocellular carcinoma," Digestive Surgery, vol. 15, no. 6, pp. 674-678, 1998.

[92] R. Izumi, K. Shimizu, T. Iyobe et al., "Postoperative adjuvant hepatic arterial infusion of lipiodol containing anticancer drugs in patients with hepatocellular carcinoma," Hepatology, vol. 20, no. 2, pp. 295-301, 1994.
[93] B. G. Peng, Q. He, J. P. Li, and F. Zhou, "Adjuvant transcatheter arterial chemoembolization improves efficacy of hepatectomy for patients with hepatocellular carcinoma and portal vein tumor thrombus," The American Journal of Surgery, vol. 198, no. 3, pp. 313-318, 2009.

[94] J. M. Llovet, S. Ricci, V. Mazzaferro et al., "Sorafenib in advanced hepatocellular carcinoma," The New England Journal of Medicine, vol. 359, no. 4, pp. 378-390, 2008.

[95] W. Y. Lau, T. W. Leung, B. S. Lai et al., "Preoperative systemic chemoimmunotherapy and sequential resection for unresectable hepatocellular carcinoma," The Annals of Surgery, vol. 233, no. 2, pp. 236-241, 2001.

[96] W. Y. Lau, S. K. Ho, S. C. Yu, E. C. Lai, C. T. Liew, and T. W. Leung, "Salvage surgery following downstaging of unresectable hepatocellular carcinoma," The Annals of Surgery, vol. 240, no. 2, pp. 299-305, 2004.

[97] L. M. Kulik, B. Atassi, L. Van Holsbeeck et al., "Yttrium90 microspheres (TheraSphere) treatment of unresectable hepatocellular carcinoma: downstaging to resection, RFA and bridge to transplantation," The Journal of Surgical Oncology, vol. 94, no. 7, pp. 572-586, 2006.

[98] S. Obi, H. Yoshida, R. Toune et al., "Combination therapy of intraarterial 5-fluorouracil and systemic interferon-alpha for advanced hepatocellular carcinoma with portal venous invasion," Cancer, vol. 106, no. 9, pp. 1990-1997, 2006.

[99] H. Nagano, A. Miyamoto, and H. Wada, "Interferonalpha and 5-fluorouracil combination therapy after palliative hepatic resection in patients with advanced hepatocellular carcinoma, portal venous tumor thrombus in the major trunk, and multiple nodules," Cancer, vol. 110, no. 11, pp. 2493-2501, 2007.

[100] L. M. Kulik, B. I. Carr, M. F. Mulcahy et al., "Safety and efficacy of Y radiotherapy for hepatocellular carcinoma with and without portal vein thrombosis," Hepatology, vol. 47, no. 1, pp. 71-81, 2008.

[101] M. R. M. Gagner and J. Dubuc, "Laparoscopic partial hepatectomy for liver tumor [Abstract].," Surgical Endoscopy, vol. 6, p. 99, 1992.

[102] K. T. Nguyen, T. C. Gamblin, and D. A. Geller, "World review of laparoscopic liver resection-2,804 patients," The Annals of Surgery, vol. 250, no. 5, pp. 831-841, 2009.

[103] A. J. Koffron, G. Auffenberg, and R. Kung, "Evaluation of 300 minimally invasive liver resections at a single institution: less is more," The Annals of Surgery, vol. 246, no. 3, pp. 385-392, 2007.

[104] A. M. Kazaryan, I. P. Marangos, A. R. Rosseland et al., "Laparoscopic liver resection for malignant and benign lesions: ten-year Norwegian single-center experience," Archives of Surgery, vol. 145, no. 1, pp. 34-40, 2010.

[105] I. Dagher, G. Belli, C. Fantini et al., "Laparoscopic hepatectomy for hepatocellular carcinoma: a european experience," The Journal of the American College of Surgeons, vol. 211, no. 1, pp. 16-23, 2010.

[106] J. Y. Cho, H. S. Han, Y. S. Yoon, and S.H Shin, "Feasibility of laparoscopic liver resection for tumors located in the posterosuperior segments of the liver, with a special reference to overcoming current limitations on tumor location," Surgery, vol. 144, no. 1, pp. 32-38, 144.

[107] Y. S. Yoon, H. S. Han, J. Y. Cho, and K. S. Ahn, "Total laparoscopic liver resection for hepatocellular carcinoma located in all segments of the liver," Surgical Endoscopy, vol. 24, no. 7, pp. 1630-1637, 2009. 
[108] J. F. Buell, D. Cherqui, and D. A. Geller, "The international position on laparoscopic liver surgery: the louisville statement," The Annals of Surgery, vol. 250, no. 5, pp. 825-830, 2009.

[109] A. Laurent, D. Cherqui, and M. Lesurtel, "Laparoscopic liver resection for subcapsular hepatocellular carcinoma complicating chronic liver disease," Archives of Surgery, vol. 138, no. 7, pp. 763-769, 2003.

[110] N. O'Rourke and G. Fielding, "Laparoscopic right hepatectomy: surgical technique," Journal of Gastrointestinal Surgery, vol. 8, no. 2, pp. 213-216, 2004.

[111] G. Belli, C. Fantini, A. D’Agostino et al., "Laparoscopic versus open liver resection for hepatocellular carcinoma in patients with histologically proven cirrhosis: short- and middle-term results," Surgical Endoscopy, vol. 21, no. 11, pp. 2004-2011, 2007.

[112] H Kaneko, M. Tsuchiya, Y. Otsuka et al., "Laparoscopic hepatectomy for hepatocellular carcinoma in cirrhotic patients," Journal of Hepato-Biliary-Pancreatic Surgery, vol. 16, no. 4, pp. 433-438, 2009.

[113] J. F. Buell, M. T. Thomas, S. Rudich et al., "Experience with more than 500 minimally invasive hepatic procedures," The Annals of Surgery, vol. 248, no. 3, pp. 475-485, 2008.

[114] L. Vigano, A. Laurent, C. Tayar, M. Tomatis, A. Ponti, and D. Cherqui, "The learning curve in laparoscopic liver resection: improved feasibility and reproducibility," The Annals of Surgery, vol. 250, no. 5, pp. 772-780, 2009.

[115] S. Todo and H. Furukawa, "Living donor liver transplantation for adult patients with hepatocellular carcinoma: experience in Japan," The Annals of Surgery, vol. 240, no. 3, pp. 451-459, (2004).

[116] S. Hwang, S. G. Lee, J. W. Joh, K. S. Suh, and D. G. Kim, "Liver transplantation for adult patients with hepatocellular carcinoma in Korea: comparison between cadaveric donor and living donor liver transplantations," Liver Transplantation, vol. 11, no. 10, pp. 1265-1272, 2005.

[117] http://www.unos.org.

[118] F. Y. Yao, L. Ferrell, N. M. Bass et al., "Liver transplantation for hepatocellular carcinoma: expansion of the tumor size limits does not adversely impact survival," Hepatology, vol. 33, no. 6, pp. 1394-1403, 2001.

[119] J. Y. Leung, A. X. Zhu, F. D. Gordon et al., "Liver transplantation outcomes for early-stage hepatocellular carcinoma: results of a multicenter study," Liver Transplantation, vol. 10, no. 11, pp. 1343-1354, 2004.

[120] J. P. Duffy, A. Vardanian, E. Benjamin et al., "Liver transplantation criteria for hepatocellular carcinoma should be expanded: a 22-year experience with 467 patients at UCLA," The Annals of Surgery, vol. 246, no. 3, pp. 502-509, 2007.

[121] Y. Sugawara, S. Tamura, and M. Makuuchi, "Living donor liver transplantation for hepatocellular carcinoma: Tokyo University series," Digestive Diseases, vol. 25, no. 4, pp. 310312, 2007.

[122] Y. Soejima, A. Taketomi, T. Yoshizumi et al., "Extended indication for living donor liver transplantation in patients with hepatocellular carcinoma," Transplantation, vol. 83, no. 7, pp. 893-899, 2007.

[123] T. Ito, Y. Takada, M. Ueda et al., "Expansion of selection criteria for patients with hepatocellular carcinoma in living donor liver transplantation," Liver Transplantation, vol. 13, no. 12, pp. 1637-1644, 2007.
[124] A. Taketomi, K. Sanefuji, Y. Soejima et al., "Impact of des-gamma-carboxy prothrombin and tumor size on the recurrence of hepatocellular carcinoma after living donor liver transplantation," Transplantation, vol. 87, no. 4, pp. 531-537, 2009.

[125] M. Berenguer, L. Ferrell, J. Watson et al., "HCV-related fibrosis progression following liver transplantation: increase in recent years," Journal of Hepato-Biliary-Pancreatic Sciences, vol. 32, no. 4, pp. 673-684, 2000.

[126] L. Lladó, J. Fabregat, J. Castellote et al., "Impact of immunosuppression without steroids on rejection and hepatitis $\mathrm{C}$ virus evolution after liver transplantation: results of a prospective randomized study," Liver Transplantation, vol. 14, no. 12, pp. 1752-1760, 2008.

[127] N. Weiler, I. Thrun, M. Hoppe-Lotichius, T. Zimmermann, I. Kraemer, and G. Otto, "Early steroid-free immunosuppression with FK506 after liver transplantation: long-term results of a prospectively randomized double-blinded trial," Transplantation, vol. 90, no. 12, pp. 1562-1566, 2010.

[128] Y. Sugawara, J. Kaneko, and M. Makuuchi, "Cyclosporin a for treatment of hepatitis C virus after liver transplantation," Transplantation, vol. 82, no. 4, pp. 579-580, 2006.

[129] Y. Sugawara, M. Makuuchi, Y. Matsui, N. Akamatsu, J. Kaneko, and N. Kokudo, "Preemptive therapy for hepatitis $\mathrm{C}$ virus after living-donor liver transplantation," Transplantation, vol. 78, no. 9, pp. 1308-1311, 2004.

[130] S. Tamura, Y. Sugawara, N. Yamashiki, J. Kaneko, N. Kokudo, and M. Makuuchi, "Pre-emptive antiviral therapy in living donor liver transplantation for hepatitis C: Observation based on a single-center experience," Transplant International, vol. 23, no. 6, pp. 580-588, 2010.

[131] J. Belghiti, A. Cortes, and E. K. Abdalla, "Resection prior to liver transplantation for hepatocellular carcinoma," The Annals of Surgery, vol. 238, no. 6, pp. 885-892, 2003.

[132] C. Margarit, A. Escartín, L. Castells, V. Vargas, E. Allende, and I. Bilbao, "Resection for hepatocellular carcinoma is a good option in Child-turcotte-pugh class a patients with cirrhosis who are eligible for liver transplantation," Liver Transplantation, vol. 11, no. 10, pp. 1242-1251, 2005.

[133] P. Majno, G Mentha, and V. Mazzaferro, "Resection, transplantation, either, or both? Other pieces of the puzzle," Liver Transplantation, vol. 11, no. 10, pp. 1177-1180, 2005.

[134] D. Cherqui, A. Laurent, N. Mocellin et al., "Liver resection for transplantable hepatocellular carcinoma: long-term survival and role of secondary liver transplantation," The Annals of Surgery, vol. 250, no. 5, pp. 738-745, 2009.

[135] Y. Takada, M. Ueda, T. Ito et al., "Living donor liver transplantation as a second-line therapeutic strategy for patients with hepatocellular carcinoma," Liver Transplantation, vol. 12, no. 6, pp. 912-919, 2006. 


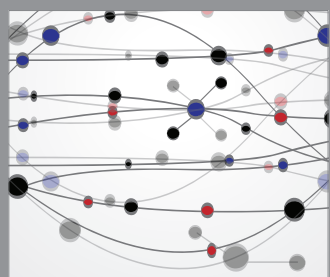

The Scientific World Journal
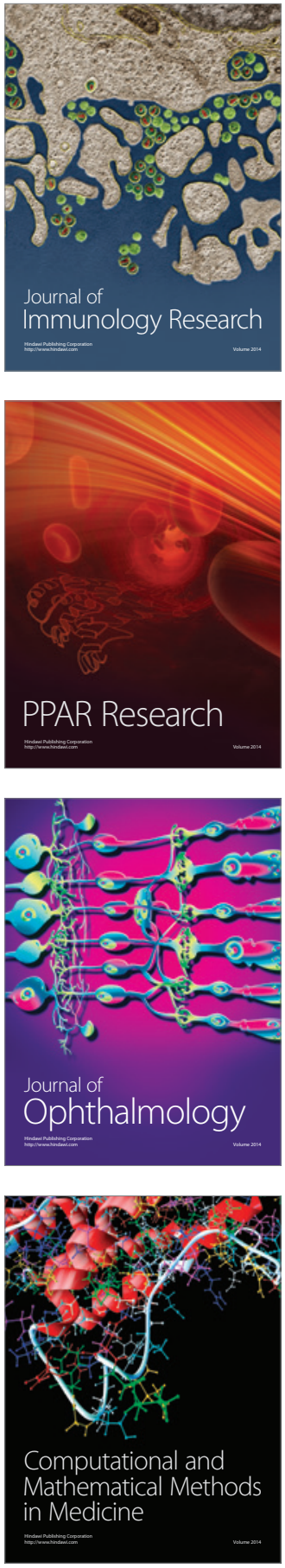

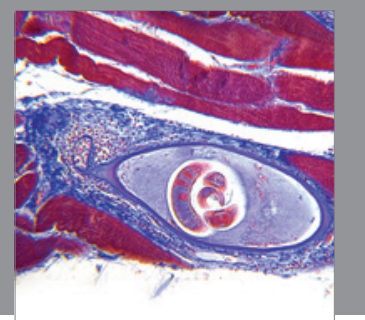

Gastroenterology

Research and Practice
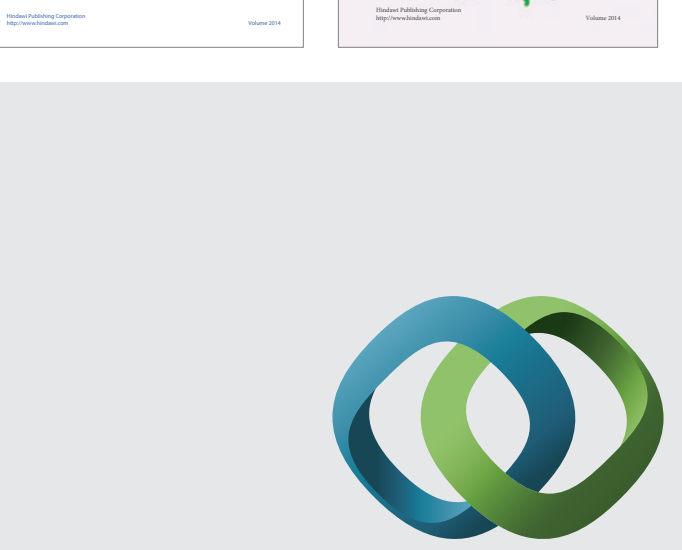

\section{Hindawi}

Submit your manuscripts at

http://www.hindawi.com
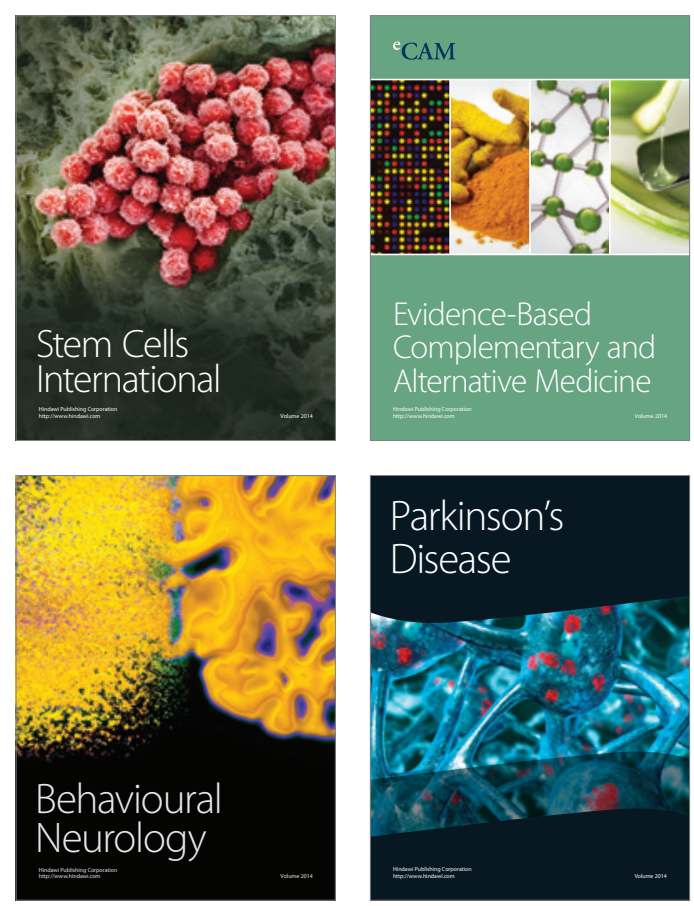

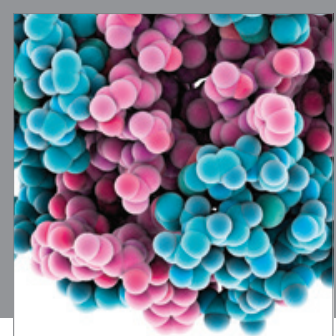

Journal of
Diabetes Research

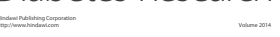

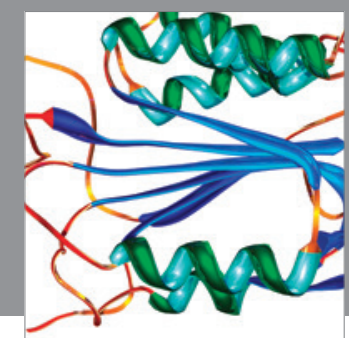

Disease Markers
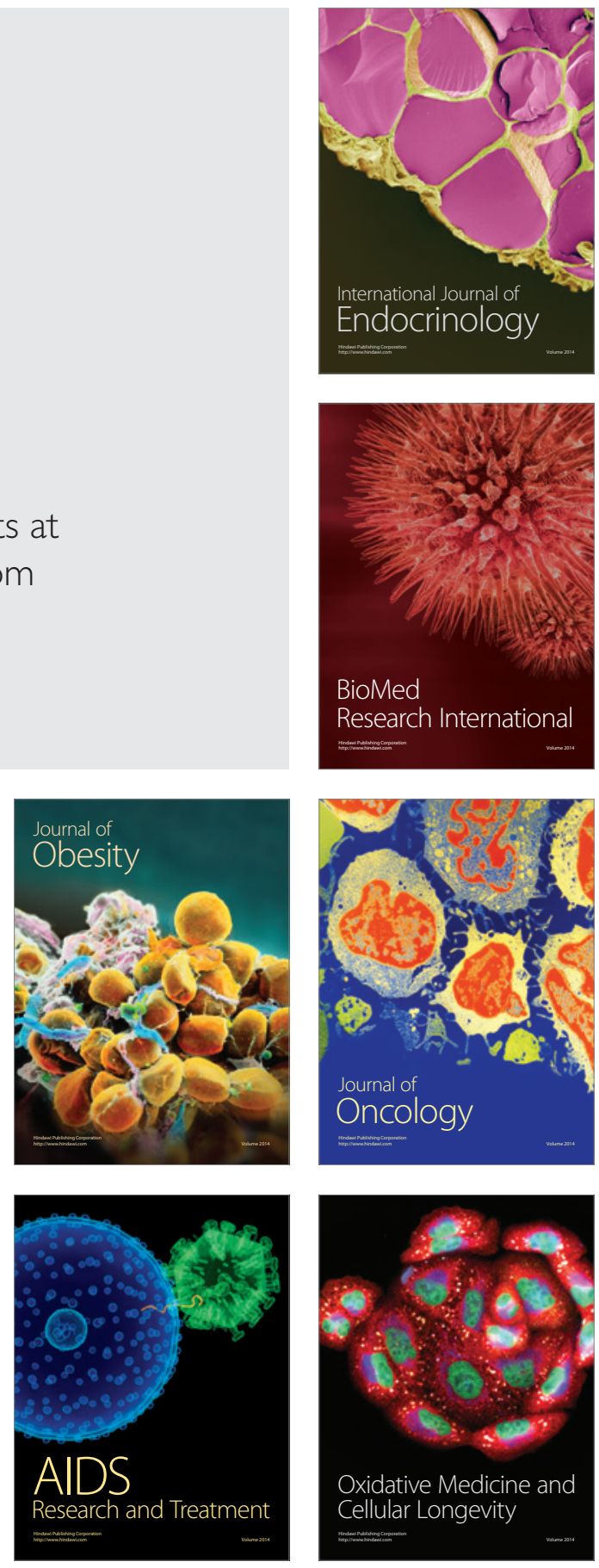